\section{(6) OPEN ACCESS}

\title{
Targeted deletion of Atg5 in chondrocytes promotes age-related osteoarthritis
}

\author{
Thibault Bouderlique, ${ }^{1}$ Karuna K Vuppalapati, ${ }^{1}$ Phillip T Newton, ${ }^{1,2}$ Lei Li, ${ }^{1}$ \\ Björn Barenius, ${ }^{3}$ Andrei S Chagin ${ }^{1}$
}

\begin{abstract}
Handling editor Tore K Kvien
- Additional material is published online only. To view please visit the journal online (http://dx.doi.org/10.1136/ annrheumdis-2015-207742).

${ }^{1}$ Department of Physiology and Pharmacology, Karolinska Institutet, Stockholm, Sweden ${ }^{2}$ Department of Women's and Children's Health, Karolinska Institutet, Stockholm, Sweden ${ }^{3}$ Orthopaedic Section, Department of Clinical Science and Education, Södersjukhuset, Karolinska Institutet, Stockholm, Sweden
\end{abstract}

\section{Correspondence to} Dr Andrei S Chagin, Bone and Cartilage Physiology Group, Department of Physiology and Pharmacology, Nanna Svartz väg2, Karolinska Institutet, Stockholm 17177, Sweden; andrei.chagin@ki.se

Received 7 April 2015 Revised 6 July 2015 Accepted 11 August 2015 Published Online First 5 October 2015

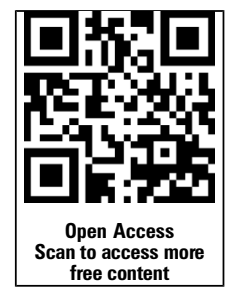

CrossMark

To cite: Bouderlique $T$ Vuppalapati KK, Newton PT, et al. Ann Rheum Dis 2016;75:627-631.

\section{ABSTRACT}

Objectives It has been suggested that the lysosomal recycling process called macro-autophagy plays a role in osteoarthritis development. We thus decided to genetically ablate the autophagy-indispensable Atg5 gene specifically in chondrocytes and analyse the development of osteoarthritis upon aging and in a post-traumatic model.

Methods Mice lacking the Atg5 gene in their chondrocytes (Atg5cKO) were generated by crossing Atg5-floxed mice with transgenic mice that expressed cre recombinase driven by the collagen type 2 promoter. Animals were analysed at the age of 2, 6 and 12 months for age-related osteoarthritis or underwent mini-open partial medial meniscectomy at 2 months of age and were analysed 1 or 2 months after surgery. We evaluated osteoarthritis using the Osteoarthritis Research Society International (OARSI) scoring on safranin-0-stained samples. Cell death was evaluated by terminal deoxy-nucleotidyl-transferase-mediated deoxyUTP nick end labelling (TUNEL) and by immunostaining of cleaved caspases.

Results We observed the development of osteoarthritis in Atg5cKO mice with aging including fibrillation and loss of proteoglycans, which was particularly severe in males. The ablation of Atg5 was associated with an increased cell death as assessed by TUNEL, cleaved caspase 3 and cleaved caspase 9 . Surprisingly, no difference in the development of post-traumatic osteoarthritis was observed between Atg5cKO and control mice.

Conclusions Autophagy protects from age-related osteoarthritis by facilitating chondrocyte survival.

\section{INTRODUCTION}

Macro-autophagy (thereafter referred as autophagy), which involves catabolic degradation of damaged organelles and long-lived protein complexes, promotes cell survival during nutritional depletion. ${ }^{1}$ This process involves phagophore formation with a double membrane, conjugation of Atg5 and Atg12 proteins, engulfment of cytosolic components and fusion of autophagosome with lysosome, where the contents are degraded and released into the cytosol. Conjugation of Atg5 and Atg12 is indispensable for autophagy. ${ }^{2}$ Ablation of Atg5 impairs autophagy and can diminish cell survival capacity. ${ }^{3}$ It was suggested that autophagy plays a role in the onset of osteoarthritis (OA). ${ }^{45}$ In healthy human and murine cartilage, autophagyrelated proteins unc-51 like autophagy activating kinase 1 (ULK1), Beclin1 and microtubule- associated protein 1A/1B-light chain 3 (LC3) are highly expressed in all the layers of articular cartilage, ${ }^{46}$ but their expression decreases during OA. ${ }^{47}$ On the other hand, in a rat model of surgically induced OA, the level of LC3-II is increased in the articular cartilage, indicating an increased autophagy. ${ }^{10}$ Mechanistic target of rapamycin complex 1 (mTORC1) intracellular signalling pathway is a master regulator of autophagy and activates autophagy when blocked. ${ }^{8}$ Inhibition of mTORC1 with either rapamycin or by targeted deletion of mTOR gene protects from surgery-induced OA in mice. ${ }^{79}$

Thus, autophagy is clearly modulated during OA development, suggesting its role in this process. However, the direct evidences are still missing. Here, we explored OA development in mice without autophagy in their chondrocytes.

\section{MATERIALS AND METHODS}

See more details in the online supplementary methods.

\section{Animal studies}

Atg $5 \mathrm{cKO}$ mice were generated by crossing Atg5floxed mice with transgenic mice that expresses cre recombinase driven by collagen type 2 promoter (Col2-Cre mice). Mice of mixed background were used for the analysis.

\section{Statistical analysis}

For all the experiments, each animal was considered as one observation. All staining were repeated five to ten times and averaged for every animal. All histological analysis was performed by 'a blinded' observer. Data are represented as mean values \pm SEM. Statistical analysis was done by unpaired Student's t test.

\section{RESULTS}

\section{Atg5 recombination in articular cartilage}

Atg5cKO (Col2-Cre;Atg5fl/fl) mice appeared normal (figure 1D) with mild growth retardation associated with elevated death of epiphyseal chondrocytes. ${ }^{11}$ First, we verified the activity of Cre recombinase in articular chondrocytes by crossing Col2-Cre mice with $\mathrm{mT} / \mathrm{mG}$ reporter mouse strain. Nearly $100 \%$ recombination was observed in the whole articular cartilage except the superficial zone (figure 1A). Next, we extracted DNA from the articular cartilage of control (Atg $5 \mathrm{fl} / \mathrm{fl}$ ) and Atg $5 \mathrm{cKO}$ mice and found recombination of Atg5 gene only in the Atg $5 \mathrm{cKO}$ cartilage (figure 1B). P62 
Figure 1 Efficiency of autophagy ablation in chondrocytes. (A) Representative photograph of the articular cartilage of $\mathrm{mT} / \mathrm{mG}$ and $\mathrm{mT} / \mathrm{mG}-\mathrm{Col} 2 \mathrm{Cre}$ mice. Upon Cre recombination, the fluorescence switches from red to green. (B) Gel showing the recombination of the Atg5 gene in the articular cartilage of Atg5cKO animals. (C) Immunostaining of P62 in the joint of control and Atg5cKO mice at 2 months of age. Bars $=100 \mu \mathrm{m}$. (D) Representative pictures of 6-month-old bones of control and Atg5cKO animals.
A

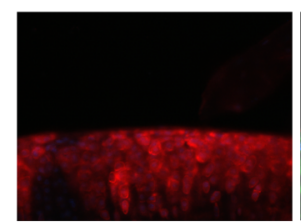

$\mathrm{mT} / \mathrm{mG}$

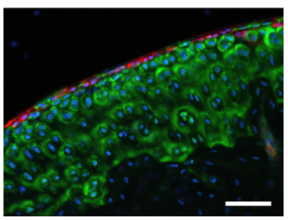

Col2-Cre;mT/mG
B

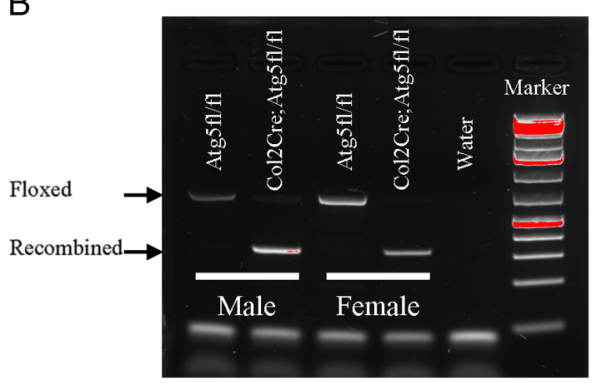

C

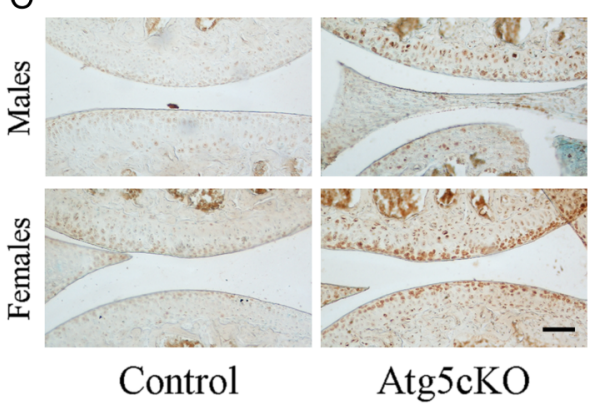

D
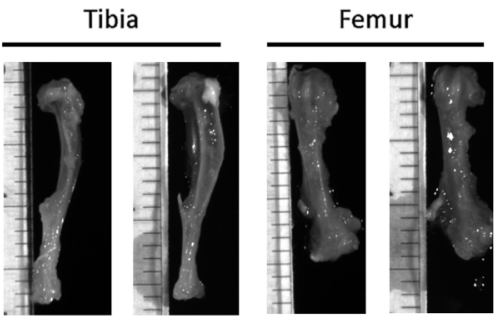

Control Atg5 $\mathrm{cKO}$ Control Atg $5 \mathrm{cKO}$ is a protein that accumulates upon autophagy inhibition and is used as a readout for autophagic activity. ${ }^{12}$ Accumulation of p62 was detected in the articular cartilage of Atg $5 \mathrm{cKO}$ males and females, but not in control (figure 1C), confirming abrogated autophagic flux. At 6 months of age, Atg5 protein was detected by western blot only in the articular cartilage of the control (see online supplementary figure S1A). Accumulation of p62 was still detected in the articular cartilage of Atg $5 \mathrm{cKO}$ mice at 6 months of age as compared with control, but less abundant than at 2 months of age (see online supplementary figure S1C).

\section{Age-related development of $O A$ in Atg5cKO mice}

At 2 months of age, both control and Atg $5 \mathrm{cKO}$ joints had no sign of joint abnormality in both genders (figure 2A). At the age of 6 months, the first signs of fibrillation and loss of proteoglycans in the superficial zone were observed in male Atg $5 \mathrm{cKO}$ joints (see figure $2 \mathrm{~A}, \mathrm{C}$ and online supplementary figure S2A). At the same time Col2-Cre; Atg5fl/+males were used as an additional control and showed no signs of OA development (data not shown). No signs of OA development were observed in Atg $5 \mathrm{cKO}$ females at 6 months of age (see figure 2A, C and online supplementary figure
A

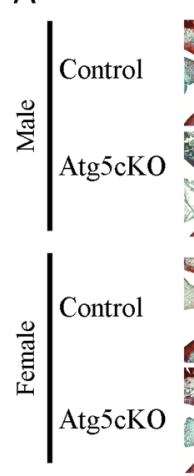

C

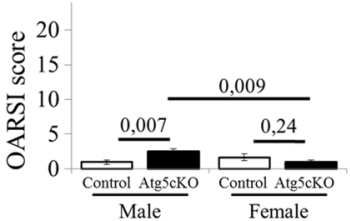

6
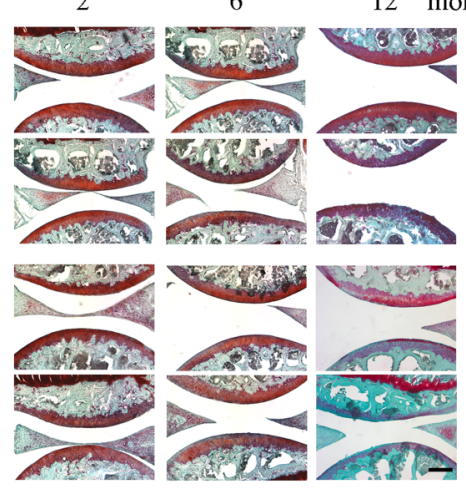

12 months

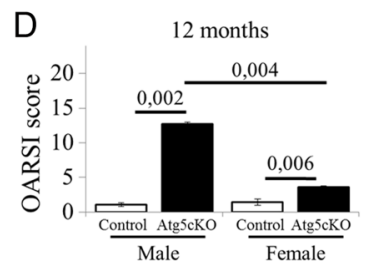

B
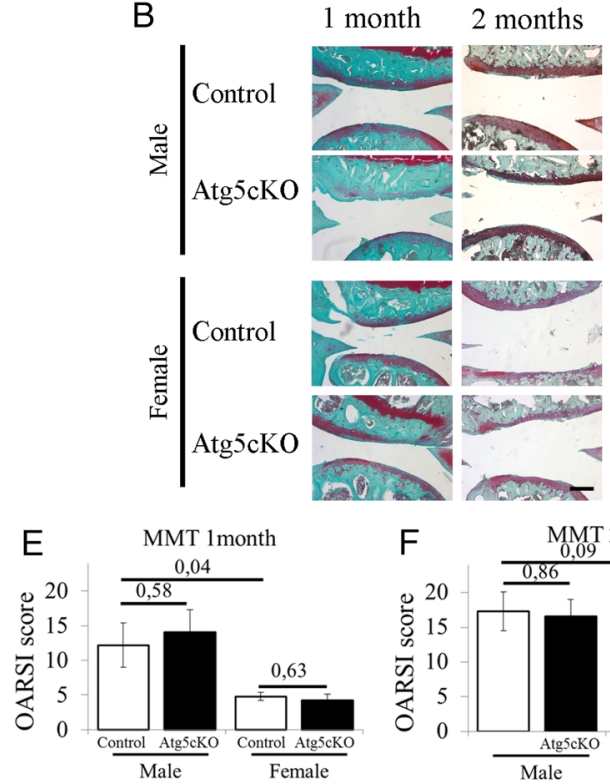

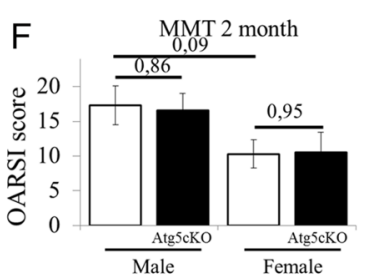

Figure 2 Histological scoring of control and Atg5cKO joints. (A) Representative photographs of the joints of male and female mice at 2, 6 and 12 months of age. (B) Representative photographs of male and female mice 1 and 2 months after partial medial meniscectomy (MMT).

Osteoarthritis Research Society International (OARSI) scoring of the joints of control and Atg5cKO male and female mice at 6 (C) and 12 (D) months of age as well as 1 (E) and 2 (F) months after surgery. Sections were stained with Safranin O/Fast green. Two-month-old: $n=6 ; 6$-month-old: males $n=5$, females $n=10$; 1 -year-old males $n=7$, females $n=10$; MMT, 1 months: $n=8,2$ months: $n=5$. Values represent mean $\pm S E M$. Bars $=200 \mu$ m. 
S2B). At 1 year of age, Atg $5 \mathrm{cKO}$ males developed substantial OA as compared with control males with fibrillation, a dramatic loss of Safranin-O staining and up to total erosion in $29 \%$ of the analysed animals (see figure 2A, D and online supplementary figure S2C). This was associated with an increase in matrix metalloproteinase (MMP)13 levels in the articular cartilage (see online supplementary figure S4C). The first signs of cartilage degradation were observed in female Atg $5 \mathrm{cKO}$ mice at this time point (figure 2AD). There was no difference in Osteoarthritis Research Society International (OARSI) score between male and female of the control group (figure $2 \mathrm{~A}, \mathrm{D}$ ), whereas Atg5 $\mathrm{cKO}$ males had a higher OARSI score than Atg5 $\mathrm{cKO}$ females (figure 2C, D). No changes were observed in the synovium, subchondral bone or formation of osteophyte (data not shown). OA develops predominantly on the medial side in humans ${ }^{13}$ although it might be different in mice. ${ }^{14}$ Accordingly, we analysed the lateral side of 1 -year-old males, but did not find any differences between control and Atg $5 \mathrm{cKO}$ (see online supplementary figure S3).

\section{Post-traumatic OA in Atg5cKO mice}

We then studied the role of autophagy in a post-traumatic model of OA. In both genders, no differences were seen
A
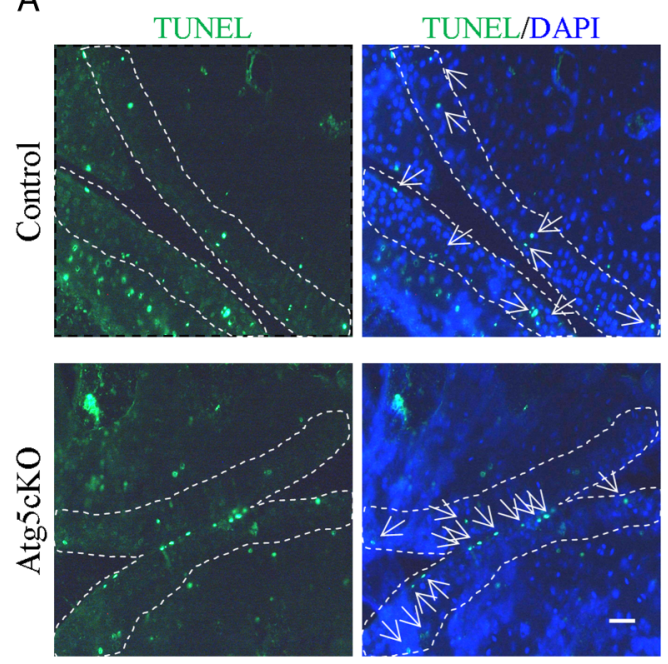

B

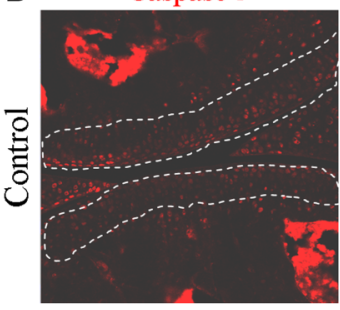

Caspase 3/DAPI
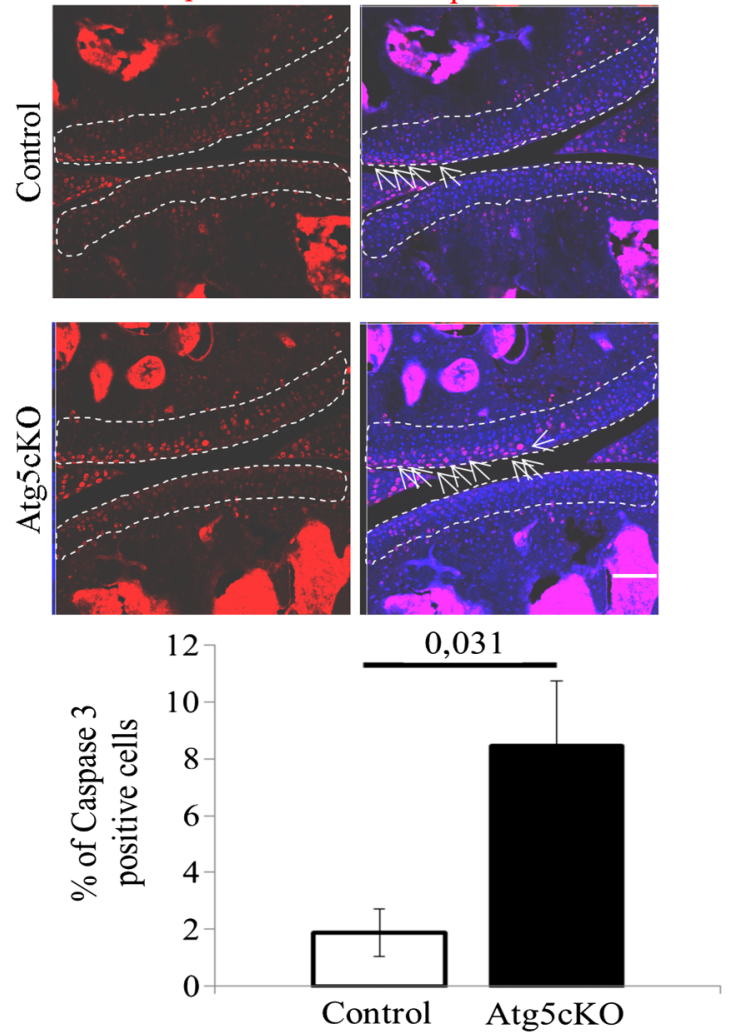
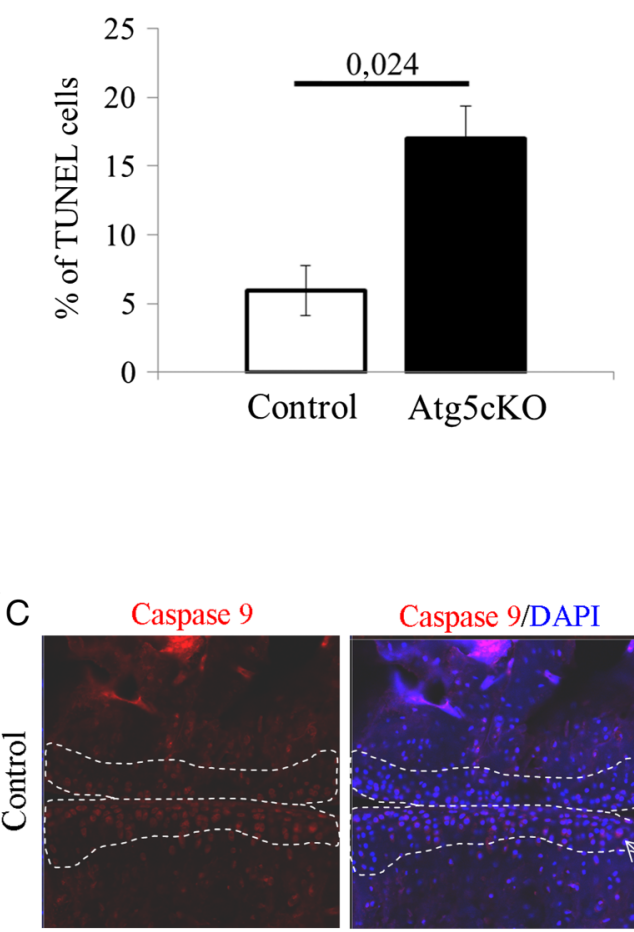

Caspase 9/DAPI
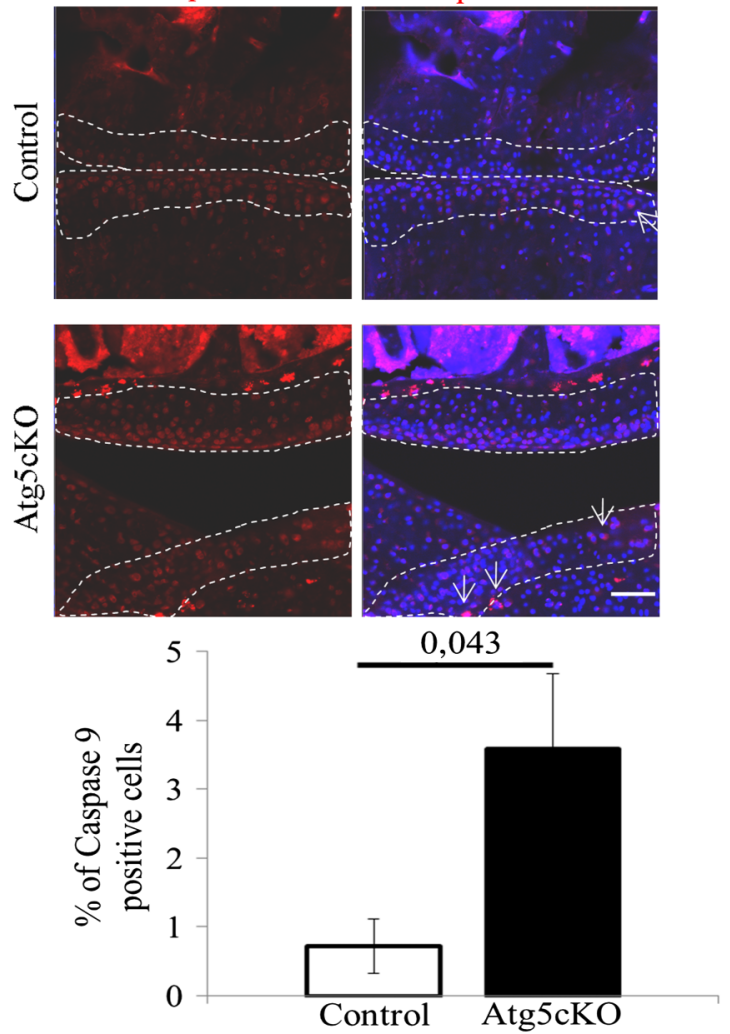

Figure 3 Cell death in the cartilage of control and Atg5cKO mice at 2 months of age. (A) Levels of terminal deoxy-nucleotidyl-transferasemediated deoxy-UTP nick end labelling (TUNEL), (B) cleaved caspase 3 and (C) cleaved caspase 9 in control and Atg5cKO articular cartilage. $n=6$ (A) and $n=5$ (B and C). Values represent mean \pm SEM. Bars $=100 \mu \mathrm{m}$. DAPI, 4', $6^{\prime}$-diamidino-2-phénylindole. 
between the control and $\operatorname{Atg} 5 \mathrm{cKO}$ animals 1 month after surgery or 2 months after surgery (see figure $2 \mathrm{~B}, \mathrm{E}, \mathrm{F}$ and online supplementary figure S2E-H). Upon surgery, males developed a more severe OA compared with females in both control and Atg $5 \mathrm{cKO}$ genotypes. No differences were observed in synovial membrane inflammation or thickening, subchondral bone sclerosis or in osteophyte formation between control and Atg5 $\mathrm{cKO}$ animals after surgery (data not shown).

\section{Atg5 ablation leads to caspase-mediated chondrocytes death}

Autophagy is a process participating in cell survival. ${ }^{3}$ Accordingly, we analysed chondrocytes apoptosis in the articular cartilage of intact 2-months-old control and Atg5cKO mice. Atg $5 \mathrm{cKO}$ mice had a higher cell death rate than control (figure $3 \mathrm{~A})$. We then assessed the level of cleaved caspase- 3 and cleaved caspase-9 in chondrocytes. Atg5cKO had a higher number of chondrocytes positive for both cleaved caspase-3 (figure 3B) and cleaved caspase-9 (figure 3D). No differences in cell death were seen at 6 months of age (see online supplementary figure S1D). No difference in cellularity was found at 2 months of age (see online supplementary figure S4A). However, the cellularity decreased at 1 year compared to 2 months and the decrease was more pronounced in Atg $5 \mathrm{cKO}$ animals (see online supplementary figure $\mathrm{S} 4 \mathrm{~A}, \mathrm{~B})$.

\section{DISCUSSION}

Autophagy genes are regulated during aging and OA development ${ }^{4}$ suggesting that autophagy is involved in this process. Our data support this idea and provide the first direct evidence that autophagy prevents cartilage degeneration in physiological setting. Genetic ablation of Atg5 leads to the development of OA with age and is associated with caspase-dependent apoptosis. Autophagy is a cell survival mechanism, especially in a low nutrient environment. ${ }^{3}$ The articular cartilage is a low nutrient tissue, ${ }^{15}$ thus impaired viability of autophagy-deficient chondrocytes might be the underlying cause of $\mathrm{OA}$ in these mice. The underlying mechanism of cell death might include release of cytochrome $C$ as we recently showed in the chondrogenic cell line RCJ 3.1C5.18. ${ }^{11}$

It is important to emphasise that cell death of articular chondrocytes is observed at 2 months of age, whereas the first signs of age-related OA become visible at 6 months. We believe that partial chondrocyte loss early in life facilitates OA development with age. Interestingly, despite the absence of Atg5, a partial restoration of p62 levels occurs at later time points, suggesting some compensatory mechanisms. We saw a similar compensation for Atg5 loss in epiphyseal chondrocytes. ${ }^{11}$ Theoretically, Atg5-independent autophagy ${ }^{16}$ or chaperone-mediated autophagy ${ }^{17}$ might compensate for Atg5 loss. This might also accounts for the absence of difference in chondrocyte death at later time points (ie, 6 months; online supplementary figure S1D). Thus, if anything, we rather underestimate the importance of autophagy for cartilage protection.

We did not observe an effect of autophagy ablation on the development of post-traumatic OA. In this model, cartilage degradation is very quick and might outweigh the mild degradation induced by ablation of autophagy. Inactivation of mTOR protects from post-traumatic OA presumably via activation of autophagy. ${ }^{7} 9$ This is in line with our general observation that autophagy has a protective function in chondrocytes although experiments with mTOR must be interpreted carefully since mTOR has numerous additional actions such as regulation of protein synthesis, microtubule organisation, lipid synthesis, cytoskeleton organisation and mitochondrial metabolism. ${ }^{8}$
Post-traumatic OA is known to be more severe in males. ${ }^{18}$ However, it was surprising to see that Atg $5 \mathrm{cKO}$ male mice are significantly more sensitive to age-related OA than female mice. It was recently reported that female mice are more sensitive to bone loss upon autophagy ablation in osteoblasts. ${ }^{19}$ On the other hand, ablation of Atg7 in osteocytes leads to more profound bone loss in males. ${ }^{20}$ Thus, there might be sex-related differences in response to autophagy ablation. The known protective effect of oestrogens on OA development ${ }^{21}$ might account for the difference between Atg $5 \mathrm{cKO}$ males and females in our study. At present, the interaction between autophagic machinery and sex hormones remains an enigma.

In summary, our data show that autophagy protects articular cartilage and the abrogation of this process leads to the development of OA upon aging, especially in male mice. This effect is likely associated with a compromised viability of the chondrocytes.

Acknowledgements The authors are grateful to Drs Camilla Svensson and Ada Delaney for their help with the surgical model of osteoarthritis.

Contributors ASC, BB and TB designed the study. TB, KKV, PTN and LL performed the experiments. TB and ASC analysed and interpreted the data. TB and ASC wrote the article. BB, KKV and PTN revised critically the manuscript. ASC and TB gave final approval to the manuscript.

Funding This work was supported by the Ulla och Gustaf af Ugglas Foundation, Karolinska Institutet and The Swedish Research Council (grant \#521-2012-1543) and The Swedish Society for Medical Research.

Competing interests None declared.

Provenance and peer review Not commissioned; externally peer reviewed.

Data sharing statement ASC and TB have access to all the data and data are available upon request.

Open Access This is an Open Access article distributed in accordance with the terms of the Creative Commons Attribution (CC BY 4.0) license, which permits others to distribute, remix, adapt and build upon this work, for commercial use, provided the original work is properly cited. See: http://creativecommons.org/ licenses/by/4.0/

\section{REFERENCES}

1 Klionsky DJ. Autophagy: from phenomenology to molecular understanding in less than a decade. Nat Rev Mol Cell Biol 2007:8:931-7.

2 Pyo JO, Nah J, Jung YK. Molecules and their functions in autophagy. Exp Mol Med 2012;44:73-80.http://www.ncbi.nlm.nih.gov/pubmed/22257882

3 Nikoletopoulou V, Markaki M, Palikaras K, et al. Crosstalk between apoptosis, necrosis and autophagy. Biochim Biophys Acta 2013;1833: 3448-59.

4 Caramés B, Taniguchi N, Otsuki S, et al. Autophagy is a protective mechanism in normal cartilage, and its aging-related loss is linked with cell death and osteoarthritis. Arthritis Rheum 2010;62:791-801.

5 Caramés B, Hasegawa A, Taniguchi N, et al. Autophagy activation by rapamycin reduces severity of experimental osteoarthritis. Ann Rheum Dis 2012;71: 575-81.

6 Yin Z. The dual role of autophagy in chondrocyte responses in the pathogenesis of articular cartilage degeneration in osteoarthritis. Int J Mol Med 2013;32: 1311-18.

7 Caramés B, Taniguchi N, Seino D, et al. Mechanical injury suppresses autophagy regulators and pharmacologic activation of autophagy results in chondroprotection. Arthritis Rheum 2012;64:1182-92.

8 Laplante M, Sabatini DM. mTOR signaling in growth control and disease. Cell 2012;149:274-93.

9 Zhang Y, Vasheghani F, Li Y-H, et al. Cartilage-specific deletion of mTOR upregulates autophagy and protects mice from osteoarthritis. Ann Rheum Dis 2015;74:1432-40.

10 Almonte-Becerril M, Navarro-Garcia F, Gonzalez-Robles A, et al. Cell death of chondrocytes is a combination between apoptosis and autophagy during the pathogenesis of Osteoarthritis within an experimental model. Apoptosis 2010;15:631-8.

11 Vuppalapati KK, Bouderlique T, Newton PT, et al. Targeted deletion of autophagy genes Atg 5 or Atg7 in the chondrocytes promotes caspase-dependent cell death and leads to mild growth retardation. J Bone Miner Res 2015. Published Online First: 15 June 2015 doi:10.1002/jbmr.2575. 
12 Klionsky DJ, Abdalla FC, Abeliovich $\mathrm{H}$, et al. Guidelines for the use and interpretation of assays for monitoring autophagy. Autophagy 2012;8:445-544. http://www.pubmedcentral.nih.gov/articlerender.fcgi?artid=3404883\&tool= pmcentrez\&rendertype =abstract (accessed $10 \mathrm{Jul}$ 2014).

13 Hunter DJ. Osteoarthritis. Best Pract Res Clin Rheumatol 2011;25:801-14.

14 Glasson SS, Chambers MG, Van Den Berg WB, et al. The OARSI histopathology initiative-recommendations for histological assessments of osteoarthritis in the mouse. Osteoarthritis Cartilage 2010;18(Suppl 3):S17-23.

15 Goldring MB, Marcu KB. Cartilage homeostasis in health and rheumatic diseases. Arthritis Res Ther 2009;11:224

16 Nishida Y, Arakawa S, Fujitani K, et al. Discovery of Atg5/Atg7-independent alternative macroautophagy. Nature 2009;461:654-8.
17 Kaushik S, Massey AC, Mizushima N, et al. Constitutive activation of chaperonemediated autophagy in cells with impaired macroautophagy. Mol Biol Cell 2008;19:2179-92.

$18 \mathrm{Ma} \mathrm{H}-\mathrm{L}$, Blanchet TJ, Peluso D, et al. Osteoarthritis severity is sex dependent in a surgical mouse model. Osteoarthritis Cartilage 2007;15:695-700.

19 Nollet M, Sabine S-D, Breuil V, et al. Autophagy in osteoblasts is involved in mineralization and bone hom...- PubMed -NCBI. http://www.ncbi.nlm.nih.gov/ pubmed/25484092 (accessed 2 Mar 2015).

20 Onal $\mathrm{M}$, Piemontese $\mathrm{M}$, Xiong J, et al. Suppression of autophagy in osteocytes mimics skeletal aging. J Biol Chem 2013;288:17432-40.

21 Martín-Millán M, Castañeda S. Estrogens, osteoarthritis and inflammation. Jt Bone Spine 2013;80:368-73. 